\title{
Gezielt verzichten - auch als Chefarzt
}

\author{
Jürg Unger-Köppel \\ Dr. med., Mitglied des FMH-Zentralvorstandes, Departementsverantwortlicher Stationäre Versorgung und Tarife
}

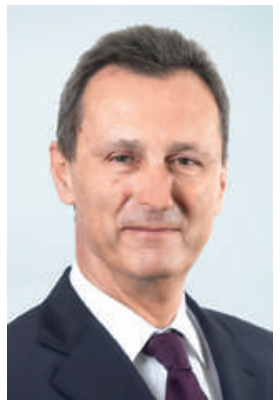

Fachlich Spitze, mit Habilitation, beste Managementqualifikationen, Leadership-Qualitäten, hervorragender Dozent, Teamplayer mit hoher Sozialkompetenz, kann begeistern, erwirbt viele Drittmittel - die Liste kann beliebig erweitert werden, wenn man Stelleninserate für Chefarztpositionen, Ordinarien oder ärztliche Direktoren liest. Sucht man die Nachfahren von Leonardo da Vinci, dem Universalgelehrten?

Es beginnt beim Numerus clausus: Selektioniert er Sozialkompetenz und Teamplayer? Lernen unsere Studierenden etwas über Management und Leadership? Mit der Facharztausbildung beherrscht man die Fachführung - aber wie steht es mit den Fähigkeiten zur Systemführung [1]? Und wann lernt man die Didaktik, die Statistik und das Publizieren? Im Studentenleben und den ersten Jahren im Beruf findet üblicherweise die Suche des Partners oder der Partnerin statt. Später folgen die Kinder. Und dann wünschen sich die Kinder, dass die Eltern zusammenbleiben und in etwa miteinander auskommen. Damit dies gelingt, braucht es genügend gemeinsame Zeit neben der Arbeit.

Viele Zielkonflikte lauern auf der Karriereleiter.

Viele Zielkonflikte kennzeichnen somit den Weg auf der Karriereleiter. Einige wenige schaffen es, diese vielfältigen Anforderungen erfolgreich zu erfüllen. Und was bleibt den anderen?

Auch in einer Zeit, in der alles möglich scheint, liegt die Lösung beim bewussten Verzicht und dem Mut zur Lücke: Studium und Facharztausbildung sind unumgänglich und bilden die wichtigste Basis für eine gute

\section{Seminarreihe Führung und Management}

Verfügen Sie über die notwendigen Instrumente, um Ihren Fachbereich oder Ihre Klinik erfolgreich im Wettbewerb zu positionieren? Aufgrund der grossen Nachfrage bietet die FMH gemeinsam mit $\mathrm{H}+$ Bildung erneut die Seminarreihe zu Führung und Management für Spitalärztinnen und Spitalärzte an. Mehr erfahren Sie unter "Seminare» auf www.fmh.ch > Stationäre Tarife.
Schweiz Ärztezeitung. 2008;89(23):1025-27. ärztliche Qualität. Sie können zunehmend in Teilzeitarbeit erfolgen, auch wenn sich damit die Ausbildungszeit verlängert und die Arbeitgeber vor organisatorische Herausforderungen gestellt werden. Günstig ist es, wenn dieser Aspekt schon bei der Wahl der Facharztausbildung berücksichtigt wird. Neben der akademischen Karriere kann man sich die Führungsthemen sehr fokussiert aneignen - die FMH bietet beispielsweise solche Seminare in Zusammenarbeit mit H+ Bildung an (siehe Kasten). Ergänzend dazu hilft ein Füh-

\section{Geeignetes Delegieren dürfte die Zufriedenheit} von Kaderärztinnen und Kaderärzten fördern.

rungscoaching. Oder man entscheidet sich gegen die Habilitation und gewinnt damit mehr Zeit für Kinder, Partner bzw. Partnerin und die eigenen Bedürfnisse und Interessen. Auch der umgekehrte Weg ist möglich. Und wenn man am Ziel angekommen ist als neu gewählte Chefärztin oder neu gewählter Chefarzt? Muss man nun alle medizinischen, administrativen und akademischen Aufgaben zusammen mit den Weiterbildungsaufgaben selbst erledigen? Auch hier hilft der bewusste Verzicht: Ein Ordinarius teilt diese Aufgaben mit einem nicht habilitierten Kollegen, der einen MBA machte. Die psychiatrische Chefärztin teilt die Aufgaben mit einer Psychologin. Zwei Chefärztinnen teilen sich die Stelle. Dies sind drei Beispiele von obersten Kaderärzten, die nicht mehr alles selbst machen, sondern sich gezielt entlasten. Nicht alle Positionen und vor allem nicht alle Vorgesetzten lassen solche Modelle zu. Oft scheut man die etwas höheren Kosten, vergisst aber, dass immer mehr Kader frühzeitig den Posten abgeben, was für die Institution sehr teuer ist. Chefärztinnen und -ärzte, die sich gezielt entlasten und Mut zur Lücke haben, Aufgaben geeignet delegieren und so neben der Arbeit noch Zeit für andere Inhalte haben, dürften zufriedener sein und länger im Amt bleiben. Das steigert die Chancen, dass sie gute, menschliche Vorgesetzte sind. Und dies alles kommt letztlich auch den Patienten sehr zu Gute. 